\title{
Challenges Experienced by Behavioral Health Organizations in New York Resulting from COVID-19: A Qualitative Analysis
}

\author{
Ann A. Murphy ${ }^{1}$ - Sean Karyczak ${ }^{1} \cdot$ Joni N. Dolce ${ }^{1} \cdot$ Michelle Zechner $^{1} \cdot$ Francine Bates $^{1} \cdot$ Kenneth J. Gill $^{1}$. \\ Pamela Rothpletz-Puglia ${ }^{2}$
}

Received: 12 August 2020 / Accepted: 16 October 2020 / Published online: 23 October 2020

(c) Springer Science+Business Media, LLC, part of Springer Nature 2020

\begin{abstract}
This paper presents a qualitative content analysis of survey data collected from behavioral health care providers from throughout New York regarding the challenges faced as a result of COVID-19. Survey responses from 295 agency and program administrators and staff, representing 238 organizations, were analyzed. Ten themes were identified: business operations, service provision, telehealth, safety, client concerns, staff concerns, supplies, technology, illness/grief/loss, and communication. These themes represent concerns that arose from the rapid transition to widespread use of telehealth, limited technology accessibility for both staff and clients, reduced revenue and billing changes, impact of COVID-19 infection itself and subsequent deaths of clients and staff, and necessary modifications for organizational communication both internally and externally. The implications of these challenges and the need for further research to identify how to best address them are discussed.
\end{abstract}

Keywords Behavioral health $\cdot$ COVID- $19 \cdot$ Telehealth $\cdot$ Service delivery $\cdot$ Content analysis

\section{Introduction}

The coronavirus disease 2019 (COVID-19) outbreak has significantly impacted the delivery of behavioral health services. Behavioral health organizations have had to rapidly modify services that are typically provided in-person, in face-to-face formats to remote delivery. As of August 2020, they are still in the process of making adaptations as the impact of the pandemic on staff and clients is still being felt.

The State of New York was one of the first epicenters of COVID-19 cases in the United States and while cases have begun to decline in New York (NY), additional states are experiencing increases (CDC 2020). Quickly after the World Health Organization (WHO) declared COVID-19 a pandemic (WHO 2020), New York issued stay at home orders effective March 20, 2020 (Chappell and Romo 2020). These

Ann A. Murphy

murphyaa@shp.rutgers.edu

1 Department of Psychiatric Rehabilitation and Counseling Professions, School of Health Professions, Rutgers, The State University of New Jersey, Piscataway, NJ, USA

2 Department of Interdisciplinary Studies, School of Health Professions, Rutgers, The State University of New Jersey, Newark, NJ, USA orders remained in effect until early June when a phased reopening process began. A full and continued reopening in New York and across the country may be hampered by the recent uptick in COVID-19 cases. The ongoing need for social distancing and the higher vulnerability of populations involved in behavioral health services may necessitate longer-term adaptations of service delivery.

People with serious mental illnesses and substance use disorders may have elevated infection rates and poorer prognoses if infected with COVID-19 due to higher rates of smoking, vaping, substance use, medical comorbidities, increased susceptibility to infections, residential instability, homelessness, limited social networks, and reduced access to medical care (Druss 2020; Kavoor 2020; Volkow 2020; Yao et al. 2020). At the same time, the stressors associated with the pandemic, including staying at home, isolation, and reduced access to recovery supports and traditional treatment, may increase the chances of exacerbating symptoms of mental illness and substance use relapse (Kavoor 2020; Volkow 2020).

Health care providers of all disciplines, including behavioral health, are also faced with very serious difficulties. Simultaneously, they are adapting to telehealth and remote delivery of services, protecting client health and safety, and managing their own health and life stressors, all in the 
context of insufficient resources (Greenberg et al. 2020). Reports of healthcare workers in China found that the added burdens related to COVID-19 were associated with increased levels of stress, depression, insomnia, and anxiety (Spoorthy et al. 2020).

Due to the recency of the pandemic, there are few reports of the specific barriers and challenges being faced by behavioral health care providers and organizations. This paper presents a qualitative content analysis of the findings of a survey completed by behavioral health care providers from throughout the State of New York regarding the challenges faced as a result of COVID-19, associated social distancing, and stay at home orders. The findings of this study can help to inform needed program and policy changes that support the delivery of high quality behavioral health services during the current and potential future pandemics, as well as other crises. A manuscript on adaptations and innovations during the pandemic is being prepared.

\section{Method}

\section{Participants}

Two hundred and ninety-five participants from 238 organizations provided complete survey data. A total of 656 organizations were invited to participate in the survey for an overall response rate of $36 \%$. One hundred and thirtyfour additional respondents began the survey but did not complete it. Incomplete responses were not included in this analysis. Fifty-one respondents (17\%) were Presidents or Chief Executive Officers (CEOs), 108 (37\%) were Associate CEOs, Vice Presidents, Senior or Division Directors, and other executives, 89 (30\%) were Program or Unit Directors, and $47(16 \%)$ were staff members below the director level. The Office of Mental Health divides New York State into five regions: western NY, central NY, Hudson River, Long Island, and New York City. Of the organizations represented in this data, 87 (37\%) were in New York City and 146 (63\%) were from other regions (17\% in western NY, $10 \%$ in central NY, 13\% in Hudson River, and 19\% in Long Island). Organizations from 44 of the 62 New York counties, including the five boroughs of New York City responded.

Agencies providing 34 of the 35 service types described in the New York State Health and Recovery Plan/Mainstream Behavioral Health Billing and Coding Manual (2017) were represented by survey respondents. Intensive Psychiatric Rehabilitation Treatment (IPRT) was the only program type not reported on by any responding participant. Twenty-five of the 35 listed service types were reported on by at least 10 responding agencies. Seventy-one responding agencies reported that they provide more than one service type (e.g., a program may provide psychosocial rehabilitation, residential support services, and supported employment). The Office of Mental Health groups service types into one of five overall categories: inpatient, emergency, outpatient, support, and residential. Among the survey respondents, 697 service types were represented and were distributed across the categories: $1 \%$ were inpatient, $2 \%$ were emergency services, $46 \%$ were outpatient services, $41 \%$ were support services, and $11 \%$ were residential services.

\section{Representativeness of Sample}

In terms of numbers of programs, as opposed to state population, there was an over-representation of responding programs from New York City and Long Island. Representation from Western NY was proportional to the actual number of programs. There was a proportional under-representation among respondents from Central NY and the Hudson Valley. In terms of the overall program categories, outpatient programs constitute only $15 \%$ of OMH related programs, but $46 \%$ of the programs who participated in the survey. Another large portion of the sample (41\%) included responses from support programs, yet this was a slight under-representation of the actual proportion of support programs $(48 \%)$. There was a slight under-representation of the state's inpatient and emergency services, and a significant under-representation of its residential programs with only $11 \%$ of respondents from residential services, while they constitute $30 \%$ of the state's programs (see Table 1).

Table 1 Representativeness of surveys received by region and $\mathrm{OMH}$ program category

\begin{tabular}{|c|c|c|}
\hline $\begin{array}{l}\text { Total \# of } \\
\text { programs }\end{array}$ & $\%$ of total & $\begin{array}{l}\text { \# par- } \\
\text { ticipating in } \\
\text { survey }\end{array}$ \\
\hline
\end{tabular}

\begin{tabular}{lllll}
\hline State region & & & & \\
New York City & 1659 & 31.6 & 259 & 37.2 \\
Hudson River & 1245 & 23.7 & 93 & 13.3 \\
Western NY & 996 & 18.9 & 119 & 17.1 \\
Central NY & 862 & 16.4 & 72 & 10.3 \\
Long Island & 496 & 9.4 & 131 & 18.8 \\
Not Reported & - & - & 23 & 3.3 \\
Total & 5258 & 100 & 697 & 100 \\
OMH program category & & & \\
Support & 2526 & 48.0 & 285 & 40.9 \\
Residential & 1581 & 30.1 & 73 & 10.5 \\
Outpatient & 785 & 14.9 & 317 & 45.5 \\
Emergency & 221 & 4.2 & 14 & 2.0 \\
Inpatient & 145 & 2.8 & 8 & 1.1 \\
Total & 5258 & 100 & 697 & 100 \\
\hline
\end{tabular}




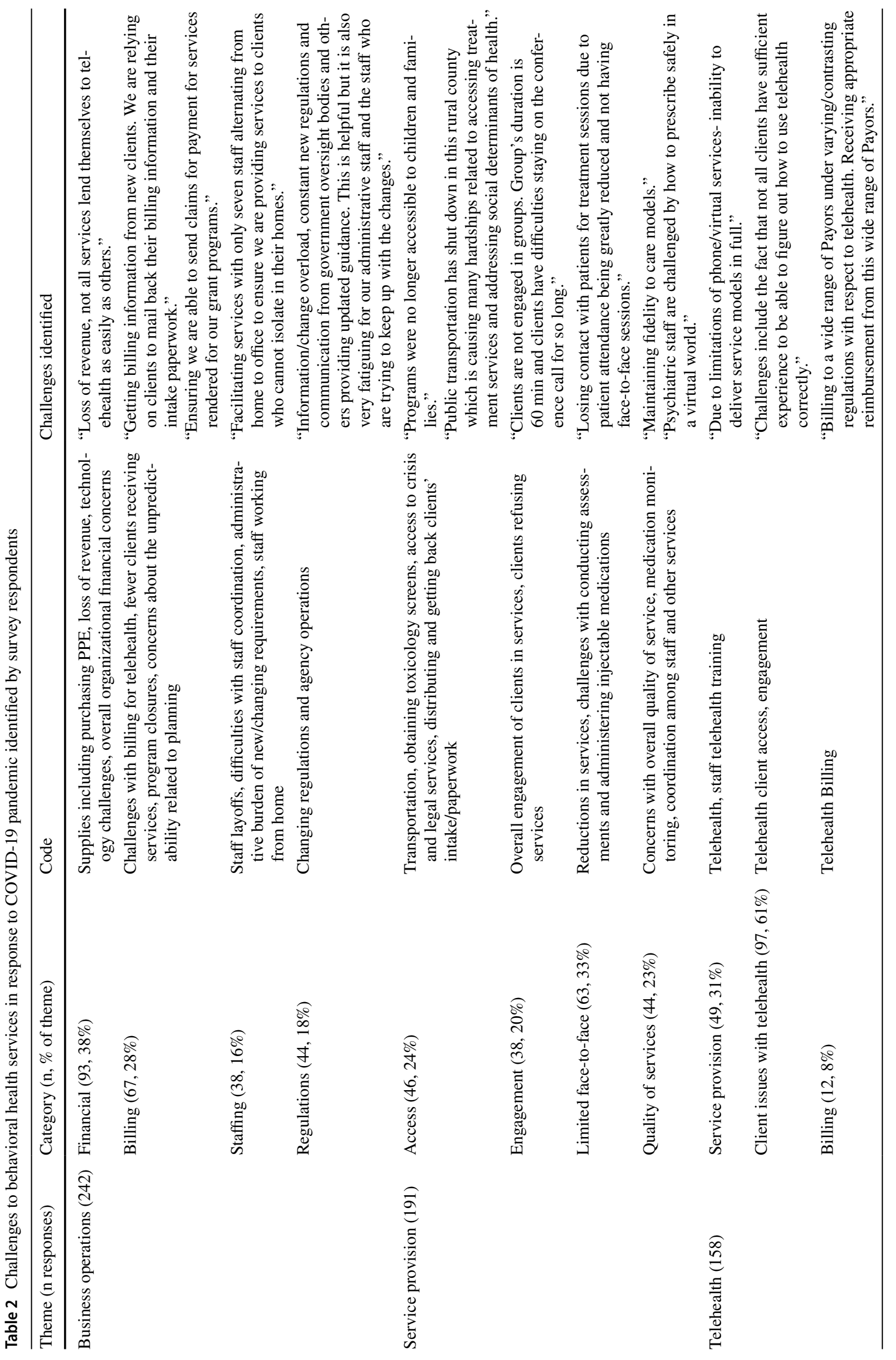




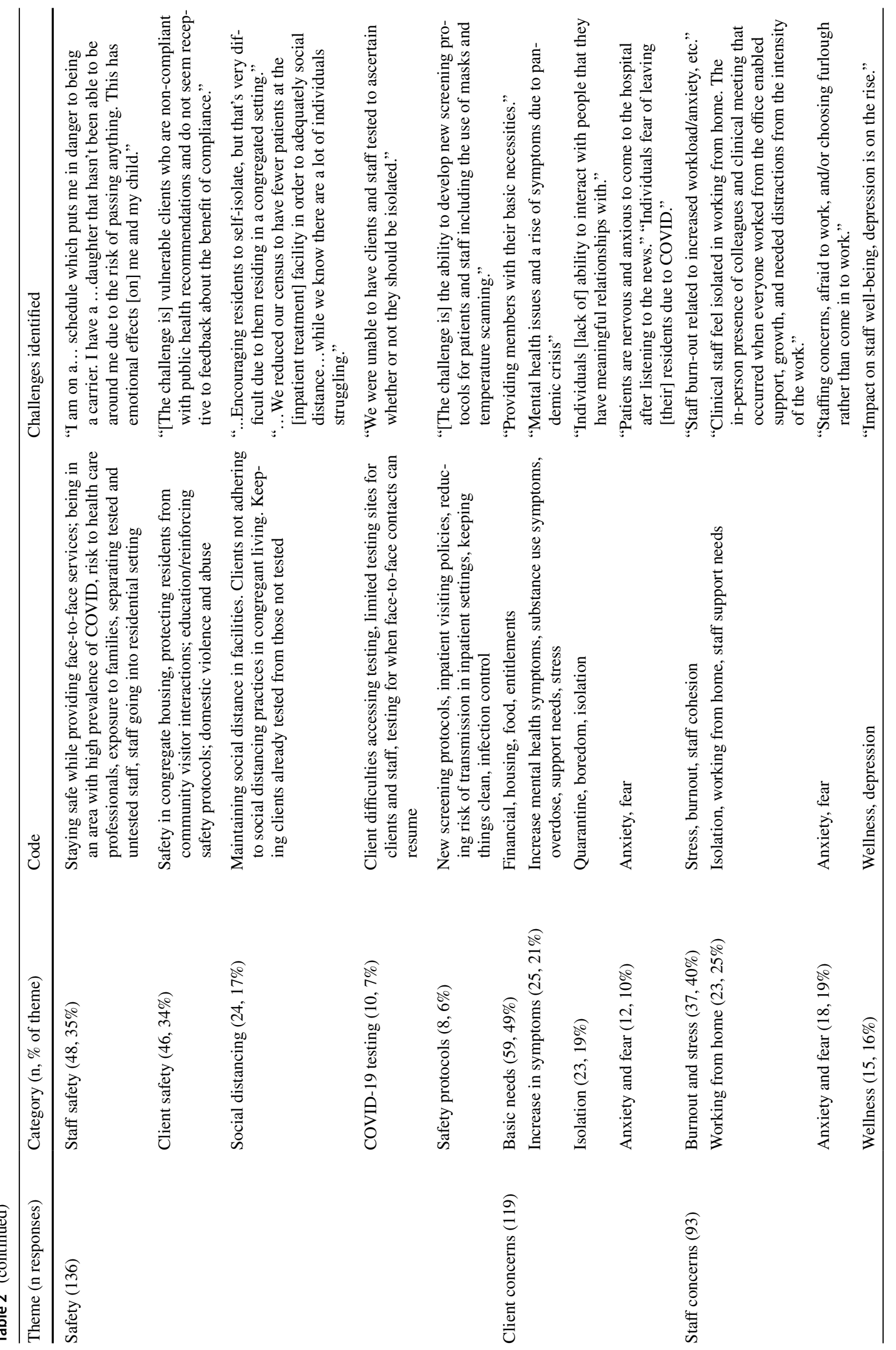




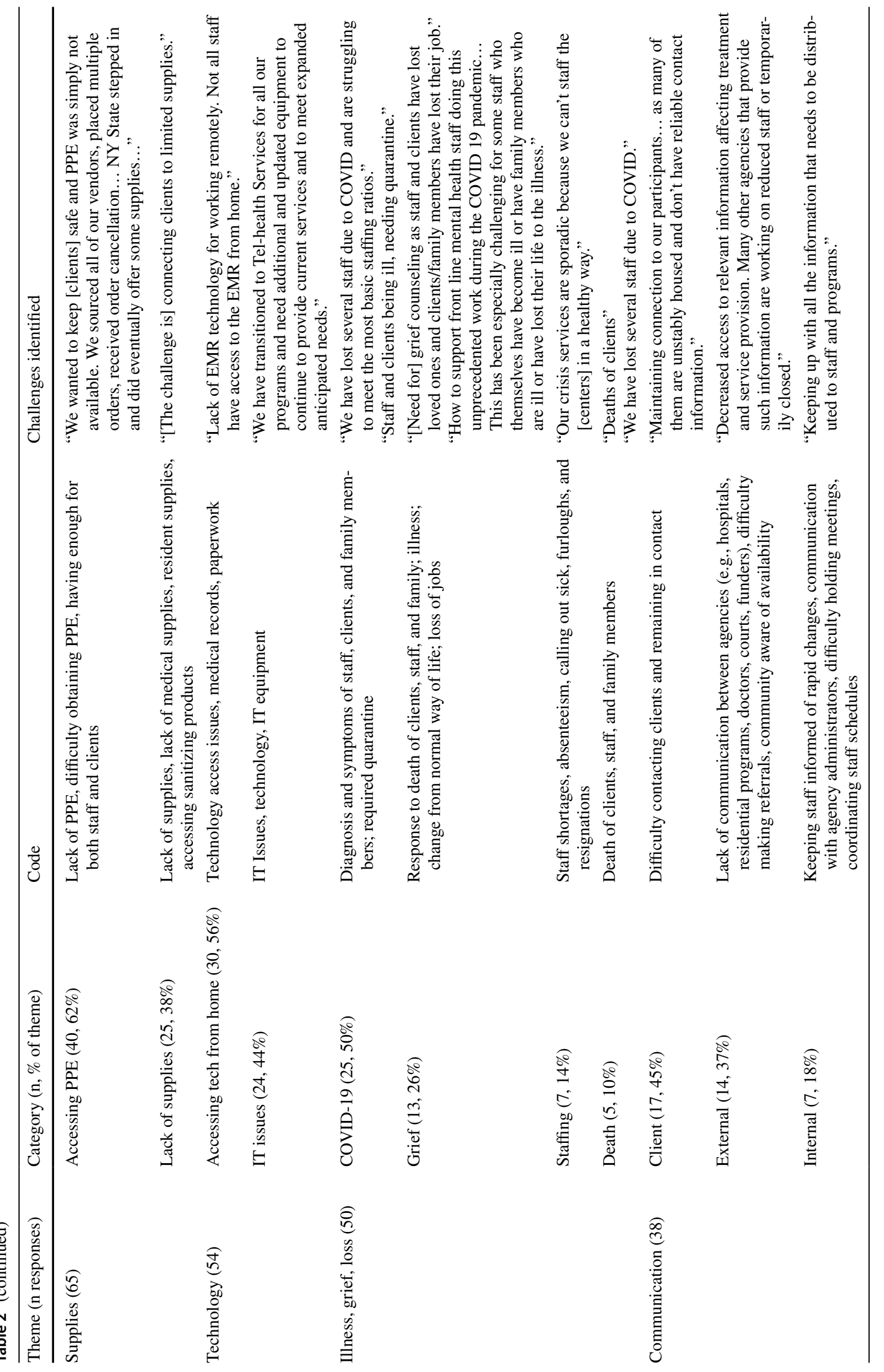




\section{Procedure}

An electronic survey was developed and distributed to behavioral health organizations throughout New York by a Substance Abuse and Mental Health Services (SAMHSA)funded center evaluation team. This survey was distributed via the project's contact list, through relevant professional organizations, the New York Office of Mental Health's provider list, and the SAMHSA regional administrator's office. The initial objective was to collect information that would aid the center in providing relevant training and technical assistance support. Following data collection and preliminary review, the authors of this paper identified data with relevance to a larger audience. Therefore, IRB approval for secondary analysis of de-identified data (Protocol 2020001516) was sought and received. De-identified data was provided from the center evaluation team to the authors.

The survey was created and administered using Qualtrics. Survey questions were developed by the SAMHSA-funded center evaluation team. Due to the novelty of COVID-19, the evaluators used exploratory open-ended survey questions to understand the challenges behavioral health care providers faced throughout the State of New York during COVID-19. The survey was opened on April 8, 2020 and closed May 1, 2020. Its questions included: participant name, contact information, name of agency/organization (identifying information was not available to authors), title in organization, identification of up to five challenges encountered due to the COVID-19 pandemic, identification of program(s) impacted by the challenge, implementation of adaptations to address the challenge, and barriers to the implementation of adaptations. This paper focuses on the analysis of the challenges identified by participants.

\section{Qualitative Content Analysis}

Qualitative data collection and analysis was used to explore and describe the phenomena of challenges to behavioral health delivery in response to COVID-19 (Palinkas et al. 2011). Given the extent of the pandemic is unparalleled in the last century, little existing theory or research literature could inform the development of specific quantitative questions. Rather it was important for the identification and description of the challenges to come from those directly experiencing them, an objective best met by open-ended qualitative questions (Hsieh and Shannon 2005). Qualitative content analysis is defined as "a research method for the subjective interpretation of the content of text data through the systematic classification process of coding and identifying themes or patterns" (p. 1278, Hsieh and Shannon 2005). The content analysis process included generating initial codes by segmenting data and labeling it with a conceptual label. Then comparisons were made among the initial codes to cluster them for the identification of patterns and a smaller number of categories. Finally, connections among the categories were identified as themes. Several strategies were undertaken to ensure the trustworthiness of the analysis (Nowell et al. 2017). The researchers conducted team coding (AM, SK, JND, MZ, and FB) and discussed disagreements until consensus was reached to enhance the reliability of the analysis. Debriefings also occurred with investigators (AM, SK, JND, MZ, FB, and KG) with content expertise. Meeting notes and reflexivity memos were maintained.

\section{Results}

Ten themes were identified from the data provided by behavioral health providers on challenges experienced because of the COVID-19 pandemic and related stay at home order. These themes represent a wide spectrum of concerns that are summarized in Table 2 and discussed in detail below.

\section{Business Operations}

Respondents identified that the transition to telehealth and remote service provision brought challenges to standard business operations. Within this theme, financial concerns were most frequently reported, including a loss of revenue due to reduction in services, increases in staff overtime pay, increased expense of purchasing personal protective equipment (PPEs) for staff and clients, and an inability to forecast the upcoming budget year. Survey respondents identified problems with reduced billing due to less service delivery and fewer clients, waiting on guidance regarding Medicaid, Medicare, and commercial insurance billing requirements, and uncertainties around reimbursement for telephonic services. Business operations were impacted by potential and immediate staff layoffs, the administrative burden of changing regulations, and coordination challenges with staff now working from home. Additionally, respondents identified problems with staffing shortages due to illness from the virus and staff attending to personal/family needs, such as caring for others in the home.

\section{Service Provision}

The most significant change in service provision was the move away from in-person, face-to-face contacts and the increased reliance on telehealth. The lack of in-person meetings made intakes, comprehensive assessments, and engagement with clients more difficult. Some clients were uninterested in engaging with staff by phone or video conference. Additionally, some clients refused remote services and others failed to answer the phone for scheduled appointments. Lack of in-person services led to reduced structure 
and activities for clients and challenges around medication monitoring and administering injectable medications.

\section{Telehealth and Technology}

The greatest number of responses related to telehealth were client access to and use of telehealth. Clients did not have, or had limited, access to technology and reliable internet services. They experienced difficulty utilizing technology to engage in services when access was available. As mentioned above, some chose not to engage using telehealth thereby reducing their overall participation in services. Many services needed to be provided by phone, as clients did not have access to computers. There were reports that clients felt overburdened by multiple calls from providers. Additionally, many clients had limited cell phone plans, curtailing their communication with providers due to inadequate minutes. For those who were able to engage in telehealth services, there were still challenges related to maintaining focus and engagement for the entirety of a planned session.

Also, staff found providing services via telehealth difficult. They highlighted their general lack of experience using both computers and telephones to provide services, as well as the rapid transition from in-person to remote services. This required staff to learn very quickly and under less than ideal circumstances how to use the technology and adapt traditional in-person services to remote delivery. Some survey respondents raised concerns that not all services were provided with a consistent level of quality when using a telehealth format. They also noted that "something was lost" by not being able to share the same physical space with a client, negatively impacting engagement and the therapeutic process.

Another challenge identified in relation to telehealth was billing for remote services. Responses indicated there was a lack of clarity regarding billing procedures and codes and changes in regulations (e.g., which requirements were being waived and which were not). The differences in regulations depending on the payor and the professional discipline were difficult to navigate. Challenges receiving appropriate reimbursement for telehealth services in a timely manner were also raised (see also Business Operations).

The heavy dependence on telehealth and the shift to working from home for many staff members was made difficult by limited internet access and insufficient bandwidth from home. A number reported lack of access to secure, HIPPA compliant platforms and an inability to access electronic medical records. Specific problems included creating forms that could be signed electronically by clients and are legally binding (e.g., consents for treatment and medication). Additionally, there was an overall lack of adequate and up to date technology offered to staff (i.e., computers, cameras, business provided cell phones, printers, scanners), as well as limited IT support to set up equipment for remote work and troubleshoot technology issues.

\section{Safety and Supplies}

Safety was a primary concern with staff safety, client safety, developing safety protocols, social distancing, and COVID-19 testing challenges identified. Shortages of PPEs, cleaning, and sanitizing products were raised. Many agencies could not obtain supplies due to a lack of availability and shipping delays. This was recognized as a threat to safety. Establishing safety measures while continuing to deliver services and addressing client needs was a high priority. Respondents noted difficulty developing, implementing, and reinforcing safety protocols to reduce the risk of virus transmission. While this was a priority everywhere, it was particularly challenging to implement social distancing protocols within residential and inpatient treatment settings. Many reduced their census to address this issue, effectively reducing access to those in need and contributing to their financial challenges from lower earned revenue. Additionally, limited access to COVID-19 testing for staff and clients made it very difficult to identify those in need of quarantine.

Related to safety, survey respondents also reported challenges regarding the need to educate clients on safety protocols and continually reinforce their importance. There were concerns about clients not understanding the seriousness of the virus and being reluctant to follow safety protocols such as social distancing and wearing face masks. Additionally, a few responses expressed the serious client safety concern of violence for those who had to remain at home with abusive family members.

\section{Client Concerns}

The greatest number of responses related to clients focused on their difficulties meeting basic needs. Food security was the top concern including the inability to meet basic nutritional needs. Much of this was financially driven due to lack of income from lost wages or disruption in entitlements. Unstable housing was also identified as a concern. Respondents reported an increase in psychiatric and substance use symptoms among clients, including increases in depression, anxiety, and overdose deaths. Understandably, clients were fearful of the pandemic and contracting the virus, leading some to refuse to attend services that remained open. Staying at home raised additional concerns regarding increased social isolation and losing connection with family, friends, and regular routines. 


\section{Staff Concerns}

Staff were concerned about the health of their colleagues and their own health. Many staff had to quickly adapt to working from home and delivering services remotely with dramatic changes in their normal working routines. All of the changes and challenges left staff feeling burned out and overworked. They worked in greater isolation and reported feeling alone, isolated, and unsupported by their coworkers who, under normal circumstances when in a shared location, helped ease their stress. Working from home removed this coping strategy. Communication and connection within teams decreased and lower morale was reported by some. For those programs that continued to provide in-person services, staff were fearful about contracting COVID-19. Some staff chose to be furloughed rather than risk infection. All of this negatively impacted staff wellness with higher rates of anxiety and depression being reported among staff.

\section{Illness, Grief and Loss}

Staff and clients testing positive, developing symptoms and dying from COVID-19 made for grim circumstances. Staff contracting the virus was a significant concern. Because of illness, some staff took significant sick leave and some had to quarantine, reducing the overall availability of personnel. Clients also experienced COVID-19 infection. This posed unique challenges for residential providers and inpatient settings to offer safe quarantine for clients testing positive, while maintaining the health of other clients and protecting staff. For providers offering case management services, the problems associated with symptomatic clients included coordinating and accessing needed care and basic communication with other health providers. Of course, respondents noted the challenges associated with the deaths of staff, clients, and their family members as a result of COVID-19. Along with grief due to client and staff death, the loss of normalcy, challenges processing grief during this time, and ensuring staff receive the support needed were also identified concerns.

\section{Communication}

Communication with clients, among staff and administrators within an organization, externally with other service providers, and with the public were all reported as problematic. Significant efforts by staff to remain in contact with clients were made, however, several respondents noted challenges with out-of-date contact information, housing instability, and technology issues (e.g. connecting via FaceTime) that made contacting clients a particular problem. Intra-agency communication was also difficult, with limited communication between administrators and staff highlighted. Rapid changes in service delivery resulted in policies and practices that were not always clearly communicated in a timely fashion. At times, disparate guidance from agency administrators also led to confusion with unclear information and mixed messages. Communication with the public and other referral agencies regarding operational status was limited initially and remained challenging. Coordinating care and communicating with insurance companies and funding agencies to clarify billing and reimbursement procedures was another concern.

\section{Discussion}

This paper offers a unique exploration of the challenges experienced by the behavioral health community during the early months of COVID-19 in the State of New York while this state was the epicenter of the pandemic in the United States. Understanding this impact is particularly important as these services support vulnerable populations that may be at higher risk for COVID-19 infection as well as other negative consequences (Druss 2020; Kavoor 2020; Volkow 2020; Yao et al. 2020). Collecting these insights via qualitative inquiry facilitated this understanding as the challenges were identified directly by those experiencing and working to address them.

Ten themes were identified: business operations, service provision, telehealth, client concerns, staff concerns, supplies, technology, illness/grief/loss, and communication. The greatest number of responses were related to business operations and largely focused on financial concerns due to the decrease in services delivered, program closures, challenges with billing for telehealth services, and increases in costs for technology and safety supplies. The prominence of this challenge in the survey results may, in part, stem from the high percentage of respondents who were in administrative positions (54\%) and therefore likely responsible for the ongoing management of business operations. If the majority of responses were from direct service providers the most reported challenge may have been different.

Many of the other challenges identified relate to the rapid transition of behavioral health services to remote delivery. This transition had to take place over a matter of days to protect the safety of clients and staff and comply with state stay at home orders and social distancing recommendations. In-person, face-to-face services quickly became telehealth services without adequate time to set up effective systems and supports. Additionally, staff lacked the proper technology and training to use the telehealth platforms. Provider organizations did not have adequate IT support to address the level of need. Clients lacked access to the necessary technology and adequate skills to utilize it. The need to rely on telehealth highlighted the 
"digital divide" that still exists for people with lower socioeconomic resources, individuals with disabilities, and for those who live in rural areas. A recent Pew Research report showed that people with disabilities, including psychiatric disabilities, are three times more likely to report never going online and about $20 \%$ less likely to own electronic devices such as tablets, smart phones, and computers or have home broadband services compared to people without disabilities (Anderson and Perrin 2017). The pandemic has underscored the need for increasing access to technology for people who are unable to take full advantage of remote and telehealth services. Thus, access to technology is another contributor to the social determinants of health (Office of Disease Prevention and Promotion, n.d.).

If the digital divide can be bridged, telehealth services may be able to provide opportunities to promote wellness and recovery more broadly. Potentially, telehealth is an effective way to reach people who have limited accessibility due to geographic location, health concerns, transportation, and time constraints (Greenbaum 2020; Perle and Nierenberg 2012). Over the past several years, prior to the pandemic, mobile mental health services have become more widely available (Greenbaum 2020; Olff 2015; Smith et al. 2020). More research needs to be conducted to assess the effectiveness of telehealth services for people in a variety of behavioral health programs. Among its limitations are inadequate access to "smart" devices for many individuals and its inability to address services, such as the dispensing of medication, toxicology screenings, comprehensive assessments, and intensive services for those with the most significant impairments-all challenges identified by our respondents.

The transition to telehealth was implemented to mitigate risk of exposure to COVID-19 for both staff and clients. Unfortunately, due to the nature of behavioral health services this was not always possible. For those in residential and inpatient services, as well as other programs that had to maintain some in-person activities, safety concerns were prominent. Efforts were made to reduce risk, but fears remained. The death of both clients and staff contributed to these fears and added grief and loss to the challenges experienced. Previous articles on COVID-19 have suggested that there is a significant emotional toll on behavioral health staff (Zhou et al. 2020). Staff are at risk of psychological distress resulting from the increased work demands, required adaptations, and anxiety and stress related to the pandemic. According to our survey, the lack of in-person interaction with colleagues and the support provided by coworkers can further exacerbate this distress. As the pandemic evolves and service delivery challenges persist, it is important to develop alternative strategies to support staff. Considerations can include increased clinical supervision, group peer supervision, staff wellness initiatives, and training and support related to technology (Bartholomew et al. 2020).
Adequate resources remain a significant need with access to PPEs, cleaning supplies, and clear safety protocols for face-to-face services still needed. Information also needs to be disseminated to clients. Education and skills instruction regarding proper hygiene, social distancing, and face coverings remains essential. Clients need to be informed about changes in services and ways to access them. Clear communication is also needed to support implementation of protocols, awareness of available services, and facilitation of service delivery. Strengthened emergency response communication plans need to be developed for the continued changes that are likely to occur during the COVID-19 pandemic and future emergency events.

While these survey findings represent a fairly comprehensive description of the challenges experienced by behavioral health providers, it is not without limitations. The data was only collected in a single state and, of course, reflects only those who voluntarily responded. This potentially limits its generalizability and representativeness. At the same time, however, numerous other geographic areas have now had to face similar challenges. Further, the State of New York is comprised of urban, suburban, and rural areas with a very diverse population potentially making their experiences more representative. In terms of within state representation, the respondents to this survey, in terms of numbers of programs, were disproportionately from outpatient services in NYC and Long Island. Not included in the present report are the many adaptations that have been implemented to meet the challenges identified which merits a separate paper and is currently in preparation.

The limited availability of technology in behavioral health agencies and the lack of skill in using technology to deliver services among staff are areas begging for research and development. As multiple respondents highlighted concerns regarding business operations and the ability to continue to remain operational, documentation of changes in service availability are needed. Indeed, the costs of not delivering these services, both in human and financial terms, must be computed.

Acknowledgements This work was support by the Substance Abuse and Mental Health Services Administration (SAMHSA) Cooperative Agreement 1H79SM081783-01.

\section{Compliance with Ethical Standards}

Conflicts of Interest The authors have no conflicts of interest to report and certify their responsibility for this manuscript.

Ethics Approval This research study was conducted utilizing previously collected data originally obtained to inform training development. Approval was obtained from the ethics committee of Rutgers Biomedical and Health Sciences Institutional Review Board for analysis of this anonymized data. The procedures used in this study adhere to the tenets of the Declaration of Helsinki. 
Informed Consent Anonymous data was provided to the authors of this manuscript for analysis and publication. As such, obtaining consent from participants is not possible.

\section{References}

Anderson, M., \& Perrin, A. (2017). Disabled Americans are less likely to use technology. Pew Research Center. https://www.pewresearc h.org/fact-tank/2017/04/07/disabled-americans-are-less-likely-touse-technology/

Bartholomew, T., Zechner, M., Birkmann, J., Reinhardt-Wood., D., Giacobbe, G., Kinter, K., \& Sperduto, J., (2020). Moving psychiatric rehabilitation workforce development and support online during COVID-19 strategies and reach. Unpublished Report: Rutgers University.

Centers for Disease Control. (2020). CDC COVID data tracker: United States COVID-19 cases and deaths by state. https://www.cdc.gov/ covid-data-tracker/\#cases

Chappell, B., \& Romo, V. (2020, June 18). New York, Illinois governors issue stay at home orders, following California's lead. National Public Radio. https://www.npr.org/sections/coronaviru s-live-updates/2020/03/20/818952589/coronavirus-n-y-govcuomo-says-100-of-workforce-must-stay-home

Druss, B. G. (2020). Addressing the COVID-19 pandemic in populations with serious mental illness. JAMA Psychiatry. https://doi. org/10.1001/jamapsychiatry.2020.0894

Greenbaum, Z. (2020). How well is telepsychology working? Monitor on Psychology, 51(5), 46-51.

Greenberg, N., Docherty, M., Gnanapragasam, S., \& Wessely, S. (2020). Managing mental health challenges faced by healthcare workers during covid-19 pandemic. British Medical Journal (Clinical Research Edition), 368, m1211. https://doi.org/10.1136/ bmj.m1211

Hsieh, H. F., \& Shannon, S. E. (2005). Three approaches to qualitative content analysis. Qualitative Health Research, 15, 1277-1288.

Kavoor, A. R. (2020). COVID-19 in people with mental illness: challenges and vulnerabilities. Asian Journal of Psychiatry, 51, 102051. https://doi.org/10.1016/j.ajp.2020.102051

New York State Health and Recovery Plan/Mainstream Behavioral Health Billing and Coding Manual. (2017). https://omh.ny.gov/ omhweb/bho/harp-mainstream-billing-manual.pdf

Nowell, L. S., Norris, J. M., White, D. E., \& Moules, N. J. (2017). Thematic analysis: striving to meet the trustworthiness criteria.
International Journal of Qualitative Methods, 16, 1-13. https:// doi.org/10.1177/I609406917733847

Office of Disease Prevention and Promotion (n.d.). Social Determinants of Health. Retrieved from https://www.healthypeople.gov/2020/ topics-objectives/topic/social-determinants-of-health.

Olff, M. (2015). Mobile mental health: a challenging research agenda. European Journal of Psychotraumatology, 6(1), 27882. https:// doi.org/10.3402/ejpt.v6.27882

Palinkas, L. A., Horwitz, S. M., Chamberlain, P., Hurlburt, M. S., \& Landsverk, J. (2011). Mixed-methods designs in mental health services research: a review. Psychiatric Services, 62, 255-263.

Perle, J., \& Nierenberg, B. (2012). How psychological telehealth can alleviate society's mental health burden: a literature review. Journal of Technology in Human Services, 31(1), 22-41. https://doi. org/10.1080/15228835.2012.760332

Smith, A., Thomas, E., Snoswell, C. L., Haydon, H., Mehratro, A., Clemensen, J., \& Caffrey, L. J. (2020). Telehealth for global emergencies: Implications for coronavirus disease 2019 (COVID-19). Telemedicine and Telecare, 26(5), 309-313.

Spoorthy, M. S., Pratapa, S. K., \& Mahant, S. (2020). Mental health problems faced by healthcare workers due to the COVID-19 pandemic: a review. Asian Journal of Psychiatry, 51, 102119. https ://doi.org/10.1016/j.ajp.2020.102119

Volkow, N. D. (2020). Collision of the COVID-19 and addiction epidemics. Annals of Internal Medicine, 173, 61-62. https://doi. org/10.7326/M20-1212

World Health Organization. (2020). WHO Director-General's Opening Remarks at the Media Briefing on COVID-19 - 11 March 2020. https://www.who.int/dg/speeches/detail/who-director-general-sopening-remarks-at-the-media-briefing-on-covid-19---11-march $-2020$

Yao, H., Chen, J. H., \& Xu, Y. F. (2020). Patients with mental health disorders in the COVID-19 epidemic. The Lancet Psychiatry, 7, e21. https://doi.org/10.1016/S2215-0366(20)30090-0

Zhou, X., Snoswell, C. L., Harding, L. E., Bambling, M., Edirippulige, S., Bai, X., \& Smith, A. C. (2020). The role of telehealth in reducing the mental health burden from COVID-19. Telemedicine and e-Health. https://doi.org/10.1089/tmj.2020.0068

Publisher's Note Springer Nature remains neutral with regard to jurisdictional claims in published maps and institutional affiliations. 